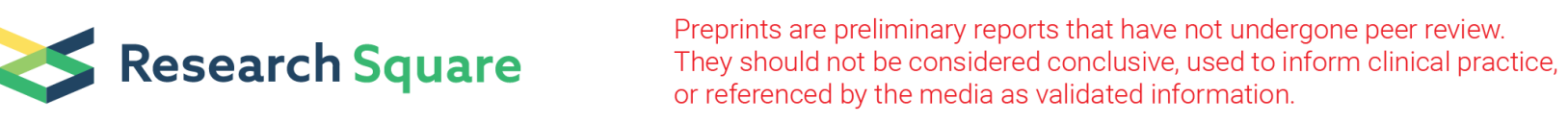

\title{
Diameter and haemodynamics of internal carotid artery siphon and ophthalmic artery in patients with non-arteritic anterior ischaemic optic neuropathy
}

Zhiyong Fu ( $\square$ fuzhiyong73@yahoo.com )

Capital Medical University https://orcid.org/0000-0002-5753-3890

Hongyang Li

Capital Medical University

Yanling Wang

Capital Medical University

\section{Research article}

Keywords: Internal carotid artery siphon, Ophthalmic artery, Blood flow volume, Non-arteritic anterior ischaemic optic neuropathy

Posted Date: September 8th, 2019

DOI: https://doi.org/10.21203/rs.2.14175/v1

License: (c) (i) This work is licensed under a Creative Commons Attribution 4.0 International License. Read Full License 


\section{Abstract}

Purpose To compare the diameter and the haemodynamics of internal carotid artery siphon(ICAS) and ophthalmic artery (OA) in patients with unilateral non-arteritic anterior ischaemic optic neuropathy (NAION) with their contralateral sides and age-matched controls.

Methods 30 patients clinically diagnosed with NAION (unilateral affected) and 30 age-matched controls participated in this study. By CTA, the diameter of ICAS and OA in both eyes were measured. By CDI, the blood flow velocity $(\mathrm{Vm})$ and the blood flow volume of ICAS and OA in both eyes were measured or calculated. Measurements on the affected side of the patients were compared to the contralateral healthy side and the controls.

Results Compared between the affected sides and the controls, the $\mathrm{Vm}$, the diameter, and the blood flow volume in the ICAS were all significantly decreased $(p<0.05)$, the $\mathrm{Vm}$ and the blood flow volume in the OA tended to be reduced ( $p=0.062,0.055$ respectively), while the difference of the diameter in the OA was insignificant ( $p=0.116)$. Compared between the affected sides and the unaffected healthy sides, the $\mathrm{Vm}$, the diameter, and the blood flow volume in the ICAS were also significantly decreased $(p<0.05)$, while no marked differences were detected in the $\mathrm{OA}$, neither for the $\mathrm{Vm}$, the diameter, or the blood flow volume $(p=0.178,0.151,0.173$ respectively $)$.

Conclusion NAION may be associated with decreased blood flow velocity and diameter in the ICAS . These findings might be potentially useful in the monitoring of NAION.

\section{Introduction}

Non-arteritic anterior ischaemic optic neuropathy (NAION) is a kind of serious ischaemic optic neuropathy, patients with NAION show a sudden, painless, decrease in vision accompanied with a visual field defect and optic disc edema[1-4]. It's believed that the pathogenesis of NAION is multifactorial[5,6], and the hypoperfusion of short posterior ciliary arteries (SPCAs)SPCAs was proved to be the major cause[7]. Previous research found that patients with NAION showed decreased velocities of blood cells in the capillaries of the optic nerve head by means of laser Doppler velocimetry[8]. Another study suggested that patients with NAION showed markedly different retrobulbar haemodynamics with reduced circulation velocity in the ophthalmic artery measured by colour Doppler imaging (CDI) [9]. As we have known, previous studies discovered a link between the pathogenesis of NAION and the intima-media thickness (IMT) IMT of carotid arteries and carotid diseases[9,10], but in our knowledge, there were few researches on the association of NAION and the diameter of ICAS so far. In fact, ICAS is a very special area from the anatomical point of view in patients with NAION[11], the narrowing of the vessel here might affect the lower reaches' hemodynamics directly.

The present study is, to our knowledge, the first of its kind to compare the diameter and the haemodynamics of ICAS and OA in patients with unilateral NAION with their contralateral sides and agematched controls. 


\section{Materials And Methods}

30 patients clinically diagnosed with NAION (unilateral affected) who presented to Beijing Friendship Hospital from July 2017 to January 2019 were selected. The mean age at diagnosis of the 30 patients was $62.1 \pm 7.3$ years (range, 47 to 72 years), and there were 18 male and 12 female. The mean interval between onset of NAION and measurement was $5.2 \pm 2.7$ days. All participants provided written informed consent.This study was approved by the local ethics committee of Beijing Friendship Hospital and was conducted in conformance with the Declaration of Helsinki (the 2013 revision), the guideline of the International Conference on Harmonisation of Good Clinical Practice.

Inclusion criteria for patients with NAION were as follows: 1) conformed to the diagnostic criteria of NAION[11], 2) unilateral optic disc swelling on ophthalmoscopy during the acute stage, 3)first time outbreak of NAION without previous treatment, and 4) completed all the examinations and follow- up visits.

Exclusion criteria were as follows: 1)any other ophthalmic diseases affecting the optic disc, including glaucoma, optic neuritis, uveitis, retinal or choroidal diseases, and trauma; and 4) any neurologic disease that may affect the optic nerve,such as multiple sclerosis, Alzheimer disease, and Parkinson disease.

The control group consisted of 30 age- matched subjects. The mean age of the 30 controls was $62.7 \pm 6.4$ years (range, 49 to 72 years), and there were 18 male and 12 female. No participant had ever had any eye disease or impaired vision function at the same period of patient recruiting. No participant had ever had cardiovascular or cerebrovascular diseases before.

All the participants were performed fundus imaging, fundus fluorescein angiography (FFA, Heidelberg, Germany) and spectral-domain optical coherence tomography scans (SD-OCT, Heidelberg, Germany) (Figure 1).

Head-and-neck computed tomographic angiography (CTA) (GE revolution CT, U.S.A) examination was performed to measure the diameter of ICAS and OA. Measurement process of the diameter of ICAS: firstly, choose the starting and the end point of ICAS segment; secondly, straighten the chosen vessel using software (GE post processing workstation AW4.6); finally, measure the narrowest part of the vessel defined as the diameter of ICAS. Measurement process of the diameter of OA: choose the vessel $5 \mathrm{~mm}$ above the level of optic canal outlet and measure the diameter of OA (Figure 2).

Color Doppler Flow Imaging (CDI) (HITCHI ALOKA, Japan) examination was employed to evaluate the hemodynamic parameters $(\mathrm{Vm})$ of ICA, with the use of linear $5-10 \mathrm{MHz}$ transducer (Figure 3). Blood flow velocities of OA were performed with a Esaote Mylab ClassC LA332 instrument (Esaote, Italy), with the use of linear 3-11 MHz transducer (Figure 4). All measurements were performed in the supine position.

The blood flow volume (A) of ICAS and OA was calculated using the following formula:

$A=\pi r^{2} \times \mathrm{Vm} \otimes r=1 / 2$ diameter $\bigotimes$ 
All the measurements were performed by experienced operators unaware of the subject's condition. The left side measurements were defined as the results of each control.

Heart rate and blood pressure (BP) were determined by sphygmomanometry in a sitting position after a rest of 5 min. Intraocular pressure (IOP) was measured before CDI examination using Goldmann applanation tonometry. Ocular perfusion pressure (OPP) was calculated using the following formula:

$\mathrm{OPP}=2 / 3($ diastolic $\mathrm{BP}+1 / 3$ (systolic $\mathrm{BP}-$ diastolic $\mathrm{BP})$ ) $-\mathrm{IOP}$

Data were expressed as mean \pm SEM. The Kolmogorov-Smirnov test was used to identify the normality of distribution. Differences in the parameters between the affected sides and the controls, and between the unaffected healthy sides and the affected sides were compared using the Mann-Whitney test, Wilcoxon test or $t$ test. $p$ value $<0.05$ was considered statistically significant. Statistical analysis was performed using the IBM SPSS software version 21.

\section{Results}

Concerned with the demographic and clinical data of the NAION patients and the controls, the age, IOP, systolic $B P$, diastolic $B P$, mean arterial pressure, heart rate and OPP showed no statistically significant difference between two groups $\llbracket p>0.05 \rrbracket$

(Table 1).

Compared between the affected sides of NAION and the controls, the diameter in the ICAS, the blood flow volume in the ICAS and the blood flow volume in the OA all significantly decreased $(p<0.05)$, while the differences of the $\mathrm{Vm}$ in the ICAS, the $\mathrm{Vm}$ in the OA and the diameter in the OA were insignificant $(\mathrm{p}=$ $0.685,0.062,0.424$ respectively) (Table 2 ).

Compared between the affected sides and the unaffected healthy sides in the patients with NAION, the difference of the Vm in the ICAS was insignificant $(p=0.652)$, while the diameter and the blood flow volume in the ICAS significantly decreased $(p<0.05)$, no marked differences were detected in the OA, neither for the $\mathrm{Vm}$, the diameter, or the blood flow volume $(p=0.261,0.197,0.094$ respectively) (Table 3).

\section{Discussion}

NAION is a major nonglaucomatous optic neuropathy, the optic nerve impairment is caused by infarction of the laminar or retrolaminar portion of the optic nerve head『which was supplied by SPCAs[6,12], the branches coming from the OA as it crosses the optic nerve. As we have known, the anatomy of SPCAs is highly variable between different individuals[13]. Since it is impossible to differentiate the individual paraoptic branches of the SPCAs and obtain the measurement data individually by using CDI[14-16], we took the hemodynamic in the $\mathrm{OA}$ as one of the subjects in the present study. Although the optic nerve 
head blood flow is only a minuscule fraction of ophthalmic artery flow[16], it is still valuable to measure the blood flow volume in the OA, for the latter could reflect the perfusion of SPCAs partly.

It's well known that small cup to disk ratio ("disk at risk") is a predisposing fator for NAION[17], furthermore, since almost all patients with acute NAION presented with papillary edema, the swelling of the nerve fibers crowded in the tight disk may compress capillaries and other small vessels among the nerve fibers [18], leading to the reduction of blood velocity of the ipsilateral central retinal artery(CRA) CRA. Therefore, CRA was not included in our study although there were studies found that the blood flow velocities of the CRA were considerably reduced in patients with acute NAION compared to healthy controls[15,19], since we didn't think the reduction of blood velocity of the ipsilateral CRA was the reason causing NAION.

ICAS was always an enigmatic region implicated in cephalic and ocular ischemic diseases. In previous studies, ICA was divided into seven segments according to Bouthillier's classification[20],they were cervical segment, petrous segment, lacerum segment, cavenous segment, clinoidal segment, ophthalmic segment, and communicating segment. The tortuous, S-shaped course(include cavenous segment and clinoidal segment) has been defined as siphon. There is a close connection between the blood flow and the morphology of vessel: the vascular bending shape would have effects on shear force of vessel wall and cause the blood flow turbulence. The tortuous configuration of ICAS is crucial to the change of blood flow parameters, which influence circulatory dynamics of blood supply; The bending shape of ICAS is also very important for diseases related to turbulent circulation[21].Therefore, we assumed that there was some link between the vessel diameter of ICAS and NAION, we wanted to verify this association in means of observing the sequentially hemodynamic changes with the intention of exploring clinical significance of ICAS.

In our study, we found the diameter of ICAS distinctly narrowed in the patients suffering from NAION, no matter compared with the unaffected healthy side or with the controls. Also, we found that the blood flow volume in the ICAS in the patients with NAION significantly decreased accordingly, although the difference of the Vm in the ICAS was insignificant compared with the unaffected healthy side and with the controls ( $p=0.652,0.685$ respectively). As we know, the resolution of CDI is insufficient up to now to provide reliable volumetric flow measurements, however, according to the law of conservation of mass, the blood flow volume should be a fixed value as blood passing through a segment of vessel, so we could aquire the instantaneous blood flow volume through the measurement of the diameter and blood flow velocities. The narrowing of the diameter of ICAS led to the reduction of blood flow volume in the ICAS, and the latter definitely diminished the volume of blood supply to the downstream region in the patients with NAION. However, previous reports proved that the degree of ICA stenosis had no significant differences between the NAION group and the controls[22-24]. From above we concluded that it was siphon but not other segments of ICA which played more important role in the occurance of NAION.

In addition to these, we noticed that the difference of $\mathrm{Vm}$ in the NAION group was insignificant compared with the unaffected healthy sides or with the controls, no matter it was the Vm of ICAS or the Vm of OA. 
According to Bernoulli's equation, the flow velocity increases as a result of a constriction or narrowing in calibre [25]. We assumed that the reason arised from this discrepancy was as follows: blood is belonged to non-Newtonian fluid, the plasma viscosity or the elasticity of vascular wall could have influence on blood flow velocity. So we thought that the blood flow velocity alone wasn't a reliable parameter in the research of NAION, but the blood flow volume seemed to be more reliable. As we know, whether the narrowing in caliber or not, the instantaneous blood flow volume was constant when blood passing though the vessel, so this parameter could reflect the situation of blood perfusion precisely, the best evidence of which was the haemodynamics of OA. The difference of diameter and $\mathrm{Vm}$ in the OA were both insignificant between the NAION group and the controls, but the OA blood flow volume reduced significantly in the affected eyes compared to the controls. Another thing worth noting is that concerned with the blood flow volume in the OA, no marked differences were detected between the affected sides and the unaffected healthy sides in the patients with NAION $(p=0.094)$. We have known that almost 25$40 \%$ patients with unilateral NAION will suffer from onset of NAION in the fellow eye afterwards, so there is reason to hypothesis that as the progress of the lesion, the blood perfusion of lower reaches might reduce furtherly and lead to the hypoperfusion of SPCAs, all the hemodynamic changes would cause hypoperfusion of the optic disc, and such ischeamic insult to the optic disc could result in NAION in the end.

we concluded that the narrowing of the diameter of ICAS and the decreased blood flow volume in the ICAS and OA may be associated with NAION. These findings might be potentially useful in the monitoring of NAION.

\section{Abbreviations}

OA ophthalmic artery

ICAS internal carotid artery siphon

NAION non-arteritic anterior ischaemic optic neuropathy

Vm the blood flow velocity

SPCAs short posterior ciliary arteries

CDI colour Doppler imaging

IMT intima-media thickness

$\mathrm{BP}$ heart rate and blood pressure

IOP intraocular pressure

OPP ocular perfusion pressure 
FFA fluorescein angiography

SD-OCT spectral-domain optical coherence tomography scans

CTA Head-and-neck computed tomographic angiography

CRA central retinal artery

\section{Declarations}

\section{Ethics approval and consent to participate}

All participants provided written informed consent.This study was approved by the local ethics committee of Beijing Friendship Hospital and was conducted in conformance with the Declaration of Helsinki (the 2013 revision), the guideline of the International Conference on Harmonisation of Good Clinical Practice.

\section{Consent for publication}

All authors have agreed to the publication of identifying images or other personal or clinical details of participants for this manuscript.

\section{Availability of data and material}

All authors have agreed to share our raw data with others, we have provided it in the supplementary file.

\section{Competing interests}

The authors have no proprietary interest in any aspect in this article.

\section{Funding}

No financial support was received.

\section{Authors' contributions}

Zhiyong Fu and Hongyang Li designed the study. Zhiyong Fu contributed to the analysis of data and drafting the manuscript and revising it critically. Hongyang Li was responsible for the interpretation of data. Yanling Wang was involved in the revising of the manuscript as well as provided final approval of the version to be published. 


\section{Acknowledgments}

The authors acknowledge Tingting Zhang, Department of Radiology, Beijing Friendship Hospital affiliated to Capital Medical University, for their assistance in collecting the data.

\section{References}

1. Hayreh SS, Zimmerman MB (2005) Visual field abnormalities in nonarteritic anterior ischemic optic neuropathy: their pattern and prevalence at initial examination. Arch Ophthalmol 123: 1554-1562

2. Johnson LN, Arnold AC (1994) Incidence of nonarteritic and arteritic anterior ischemic optic neuropathy. Population-based study in the state of Missouri and Los Angeles County, California. $J$ Neuroophthalmol 14: 38-44

3. Hata M, Oishi A, Muraoka Y, Miyamoto K, Kawai K, Yokota S, Fujimoto M, Miyata M, Yoshimura N (2017) Structural and functional analyses in nonarteritic anterior ischemic optic neuropathy: optical coherence tomography angiography study. J Neuroophthalmol 37: 140-148

4. Gaier ED, Torun N (2016) The enigma of nonarteritic anterior ischemic optic neuropathy: an update for the comprehensive ophthalmologist. Curr Opin Ophthalmol 27: 498-504

5. Hayreh SS (2000) Ischaemic optic neuropathy. Indian J Ophthalmol 48:171-94

6. Arnold AC (2003) Pathogenesis of nonarteritic anterior ischemic optic neuropathy. J Neuroophthalmol 23:157-163

7. Arnold AC (2016) The 14th Hoyt lecture: ischemic optic neuropathy: the evolving profile,1966-2015. J Neuroophthalmol 36: 208-215

8. Collignon-Robe NJ, Feke GT, Rizzo JF(2004) Optic nerve head circulation in nonarteritic anterior ischemic optic neuropathy and optic neuritis. Ophthalmol 111:1663-72

9. Sanjari MS, Falavarjani KG, Mehrabani M, Ghiasian L, Zamani B (2009) Retrobulbar haemodynamics and carotid wall thickness in patients with non-arteritic anterior ischaemic optic neuropathy. $\mathrm{Br} \mathrm{J}$ Ophthalmol 93: 638-640

10. Horowitz J, Melamud A, Sela L, Hod Y, Geyer O (2001) Internal carotid artery hypoplasia presenting as anterior ischemic optic neuropathy. Am J Ophthalmol 131:673-674

11. Singh R, Tubbs RS (2017) Effect of cervical siphon of external and internal carotid arteries. $J$ Craniofac Surg 28: 1857-1860

12. Mayes EW, Cole ED, Dang S, Novais EA, Vuong L, Mendoza-Santiesteban C, Duker JS, Hedges TR. 3rd (2017) Optical coherence tomography angiography in nonarteritic anterior ischemic optic neuropathy," J Neuroophthalmol 37: 358-364

13. Olver JM, Spalton DJ, McCartney AC (1994) Quantitative morphology of human retrolaminar optic nerve vasculature. Invest Ophthalmol Vis Sci 35:3858-3866

14. Hayreh SS (2001) The blood supply of the optic nerve head and the evaluation of it-myth and reality. Prog Retin Eye Res 20: 563-593 
15. 15 Zhu WH, Cui MY, Yao FJ, Liao RD, Liu L (2014) Retrobulbar and common carotid artery haemodynamics and carotid wall thickness in patients with non-arteritic anterior ischaemic optic neuropathy. Graefes Arch Clin Exp Ophthalmol 252:1141-1146

16. Feke GT (2006) Retrobulbar haemodynamics in non-arteritic anterior ischaemic optic neuropathy. $\mathrm{Br}$ J Ophthalmol 90:1334-1335

17. Atkins EJ, Bruce BB, Newman NJ, Biousse V (2010) Treatment of nonarteritic anterior ischemic optic neuropathy. SurvOphthalmol 55: 47-63

18. Hayreh SS, Zimmerman MB (2007) Optic disc edema in non-arteritic anterior ischemic optic neuropathy. Graefes Arch Clin Exp Ophthalmol 245:1107-1121

19. Kaup M, Plange N, Arend KO, Remky A (2006) Retrobulbar hemodynamics in non-arteritic anterior ischemic optic neuropathy. $\mathrm{Br} \mathrm{J}$ Ophthalmol 90:1350-1353

20. Bouthillier A, van Loveren HR, Keller JT (1996) Segments of the internal carotid artery: a new classification. J Neurosurg 38:425-433

21. Zenteno M, Vinuela F, Moscote-Salazar LR, Alvis-Miranda H, Zavaleta R, Flores A, Rojas A, Lee A (2014) Clinical implications of internal carotid artery tortuosity, kinking and coiling: a systematic review. Rom Neurosurg21:50-59

22. Fry CL, Carter JE, Kanter MC, Tegeler CH, Tuley MR (1993) Anterior ischemic optic neuropathy is not associated with carotid artery atherosclerosis. Stroke 24:539-542

23. Muller M, Kessler C, Wessel K, Mehdorn E, Kompf D (1993) Low-tension glaucoma: a comparative study with retinal ischemic syndromes and anterior ischemic optic neuropathy. Ophthalmic Surg Las 24: $835-838$

24. McCullough HK, Reinert CG, Hynan LS, Albiston CL, Inman MH, Boyd PI, Welborn MB. 3rd., Clagett GP, Modrall JG (2004) Ocular findings as predictors of carotid artery occlusive disease: is carotid imaging justified? J Vasc Surg 40:279-286

25. KagemannL, HarrisA, ChungHSetal (1999) Basicsandlimitations of CDI. In: Pillunat LE, Harris A, Anderson DR, Greve EL (eds) Current concepts on ocular blood flow in glaucoma. Kugler Publications, The Hague, pp 103-110

\section{Tables}

Table 1 Demographic and clinical data of the patients with NAION and controls 


\begin{tabular}{cccc}
\hline & NAION & Controls & $P$ value \\
\hline Age (years) & $62 \pm 7$ & $63 \pm 6$ & $0.765^{*}$ \\
\hline IOP (mm Hg) & $15 \pm 3$ & $14 \pm 3$ & $0.607^{*}$ \\
Systolic BP & $140 \pm 26$ & $132 \pm 19$ & $0.193^{*}$ \\
(mm Hg) & & & \\
Diastolic BP & & & \\
(mm Hg) & $79 \pm 14$ & $74 \pm 11$ & $0.120^{*}$ \\
Mean arterial pressure (mm Hg) & $99 \pm 17$ & $93 \pm 13$ & $0.141^{*}$ \\
Heart rate (per min) & $75 \pm 9$ & $74 \pm 11$ & $0.686^{*}$ \\
OPP (mm Hg) & $52 \pm 10$ & $48 \pm 8$ & $0.133^{*}$ \\
\hline
\end{tabular}

Table 2 Comparison of diameter, Vm and blood flow volume in the OA and ICAS between the affected side of the patients with NAION and the controls

\begin{tabular}{cccc}
\hline & NAION eye & Controls & $P$ value \\
\hline OA diameter $(\mathrm{mm})$ & $1.37 \pm 0.10$ & $1.40 \pm 0.13$ & $0.424^{* *}$ \\
OA Vm $(\mathrm{cm} / \mathrm{s})$ & $11.61 \pm 2.79$ & $12.76 \pm 1.72$ & $0.062^{*}$ \\
OA blood flow volume $\left(\mathrm{mm}^{3} / \mathrm{s}\right)$ & $172.50 \pm 52.50$ & $198.42 \pm 39.41$ & $0.035^{*}$ \\
ICAS diameter $(\mathrm{mm})$ & $2.97 \pm 0.75$ & $3.39 \pm 0.60$ & $0.022^{*}$ \\
ICAS Vm $(\mathrm{cm} / \mathrm{s})$ & $45.92 \pm 6.66$ & $46.53 \pm 4.68$ & $0.685^{*}$ \\
ICAS blood flow volume $\left(\mathrm{mm}^{3} / \mathrm{s}\right)$ & $3212.67 \pm 1128.37$ & $4209.33 \pm 1177.73$ & $0.001^{*}$ \\
\hline
\end{tabular}

Table 3 Comparison of diameter, Vm and blood flow volume in the OA and ICAS between the affected and unaffected sides in the patients with NAION 


\begin{tabular}{cccc}
\hline & NAION eye & Nomal eye & $P$ value \\
\hline OA diameter $(\mathrm{mm})$ & $1.37 \pm 0.10$ & $1.39 \pm 0.12$ & $0.197^{* * *}$ \\
OA Vm $(\mathrm{cm} / \mathrm{s})$ & $11.61 \pm 2.79$ & $12.11 \pm 3.11$ & $0.261^{*}$ \\
OA blood flow volume $\left(\mathrm{mm}^{3} / \mathrm{s}\right)$ & $172.50 \pm 52.50$ & $186.37 \pm 61.41$ & $0.094^{*}$ \\
ICAS diameter $(\mathrm{mm})$ & $2.97 \pm 0.75$ & $3.21 \pm 0.57$ & $0.015^{*}$ \\
ICAS Vm $(\mathrm{cm} / \mathrm{s})$ & $45.92 \pm 6.66$ & $45.33 \pm 6.00$ & $0.652^{*}$ \\
ICAS blood flow volume $\left(\mathrm{mm}^{3} / \mathrm{s}\right)$ & $3212.67 \pm 1128.37$ & $3721.75 \pm 1184.39$ & $0.005^{*}$ \\
\hline
\end{tabular}

Data are mean \pm SEM , t test*, Mann-Whitney test**, Wilcoxon test $* * *$

$N A I O N$ non-arteritic anterior ischaemic optic neuropathy; $O A$ ophthalmic artery; $V m$ the blood flow velocity; ICAS internal carotid artery siphon

\section{Figures}
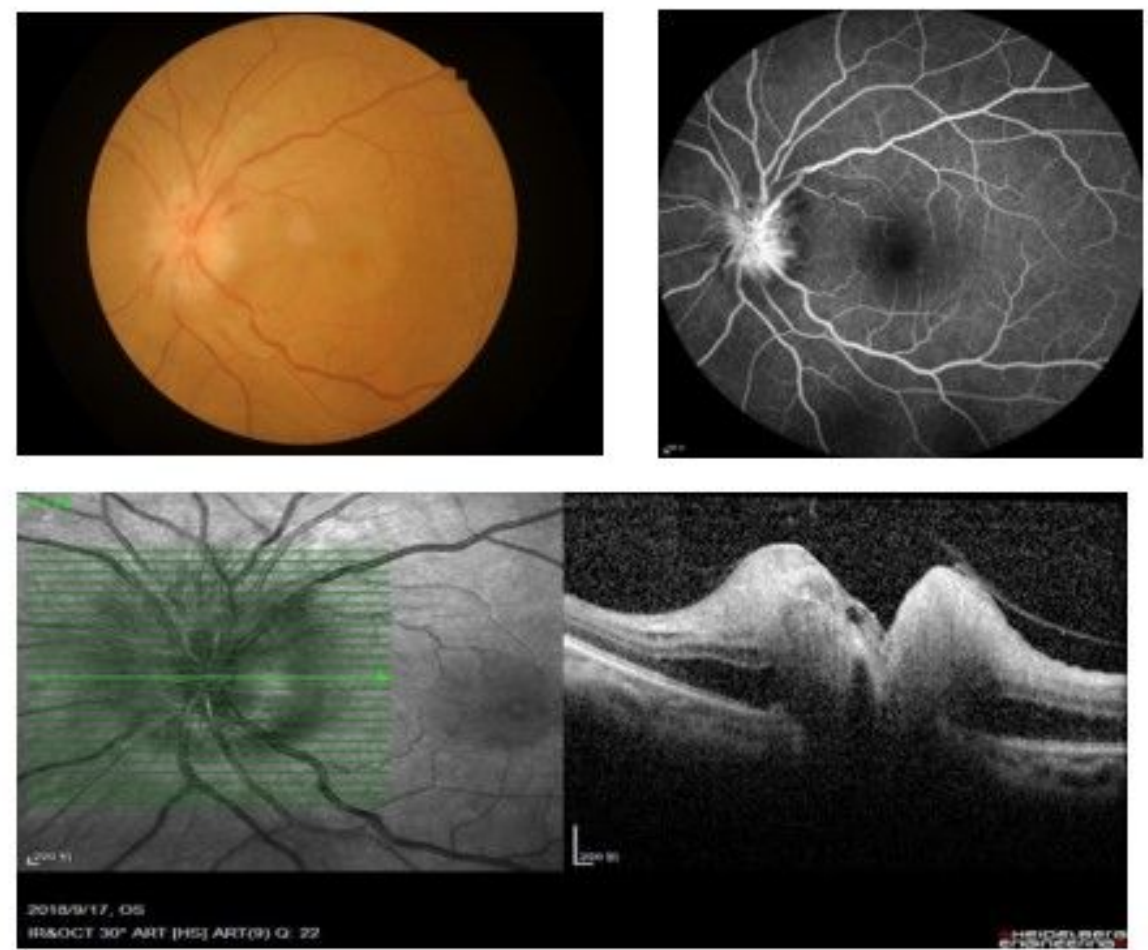

\section{Figure 1}

Fundus imaging. FFA and OCT showed the optic disc swollen and optic papilla surface fluorescence leakage. 

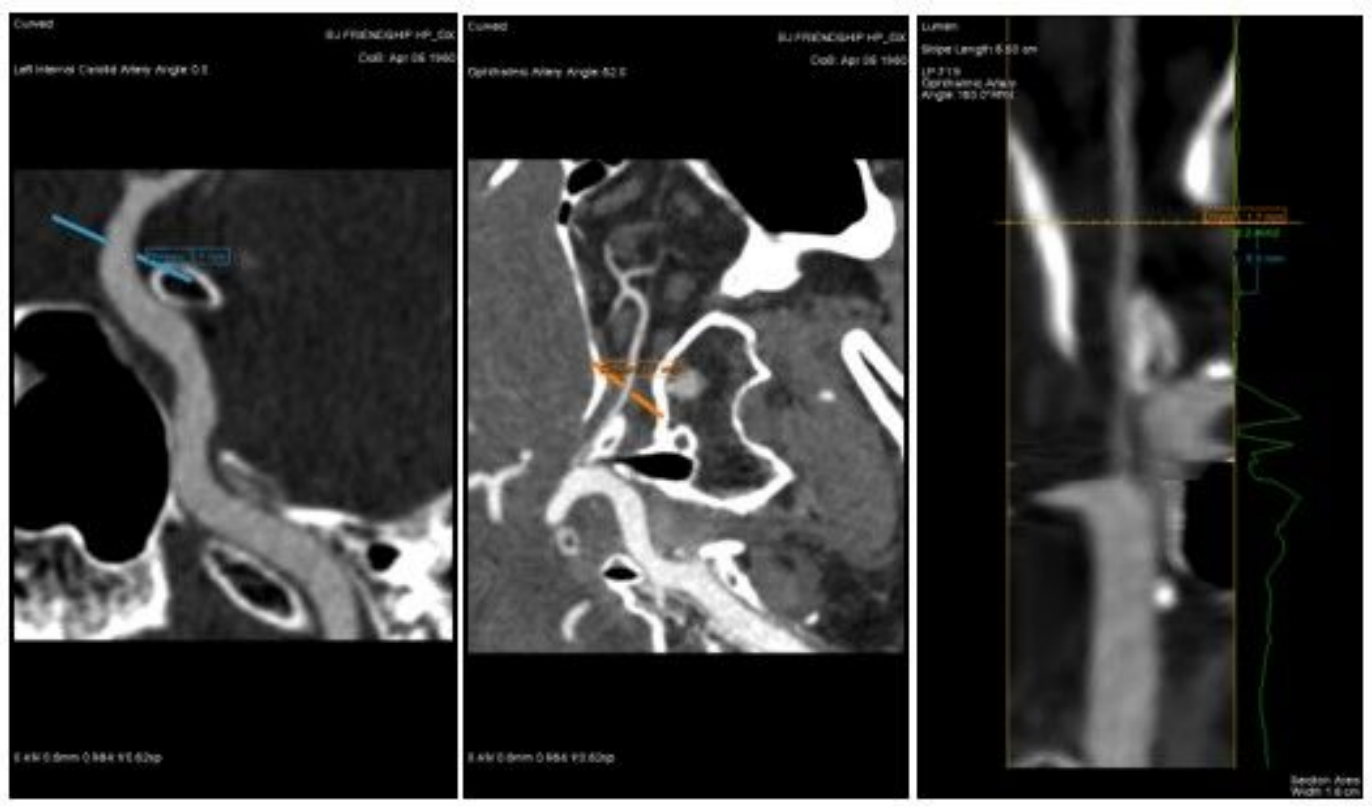

Figure 2

Head-and-neck CTA inspection. Showed the diameter of ICAS and OA.

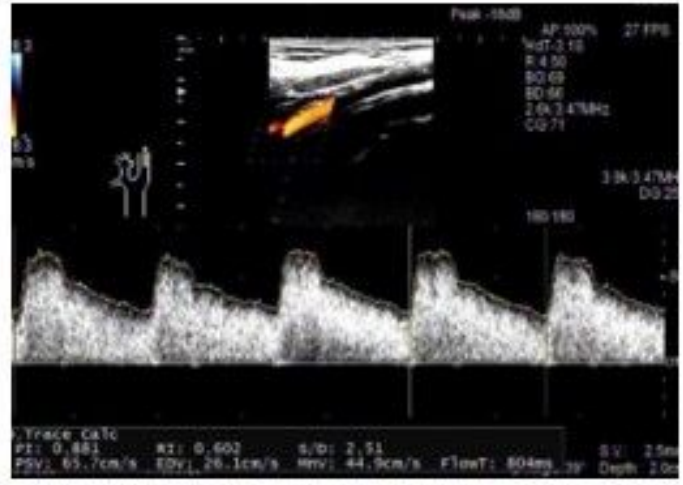

Figure 3

CDI examinations of ICAS. Showed the haemodynamics of ICAS.

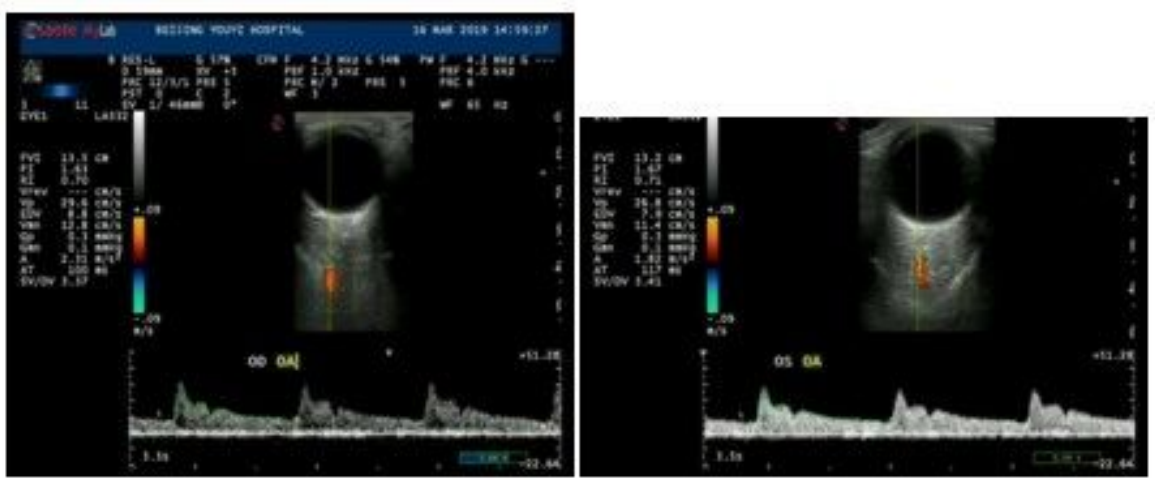

Figure 4 
CDI examinations of OA. Showed the haemodynamics of OA.

\section{Supplementary Files}

This is a list of supplementary files associated with this preprint. Click to download.

- NAIONdata.xls 